\title{
Faden Operation (Posterior Fixation Sutures)
}

\author{
BRIAN HARCOURT $\dagger$
}

Leeds

The ocular rotational power which a rectus muscle exerts is a product of the muscle's force and the length of the lever arm between the muscle's tangential point of contact with the globe and the centre of rotation of the eye.

A recession operation weakens a rectus muscle because the distance between the muscle's origin at the orbital apex and its insertion into the surface of the globe is decreased, so that the muscle becomes slack. Provided that the recession does not exceed the conventional maximum (Table I), the length of the lever arm is not reduced. Even when the eye moves into the field of action of the recessed muscle, the length of the rotational lever arm remains unchanged as the tangential point of muscle action is still on the surface of the globe. If the muscle is overrecessed, not only does it become very slack but the effective lever arm is reduced in length and mechanical restriction of movement ensues. The weakening effect of a conventional recession operation is therefore approximately the same in all directions of gaze in which the muscle is active (Figs. 1 and 2).

Table I Maximum recession possible without limiting ocular motility.

\begin{tabular}{lr}
\hline Superior rectus & $5 \mathrm{~mm}$ \\
Inferior rectus & $5 \mathrm{~mm}$ \\
Medial rectus & $5.5 \mathrm{~mm}$ \\
Lateral rectus & $8 \mathrm{~mm}$ \\
\hline
\end{tabular}

The faden operation was first described by Adelstein and Cüppers. The word derives from the German for a thread or suture; the more descriptive English term is posterior fixation suture. The operation aims to produce a weakening which is proportionately less than that of an equivalent recession when the eye is in the straightahead position, but which increases progressively as the eye moves into the field of action of the operated muscle. This
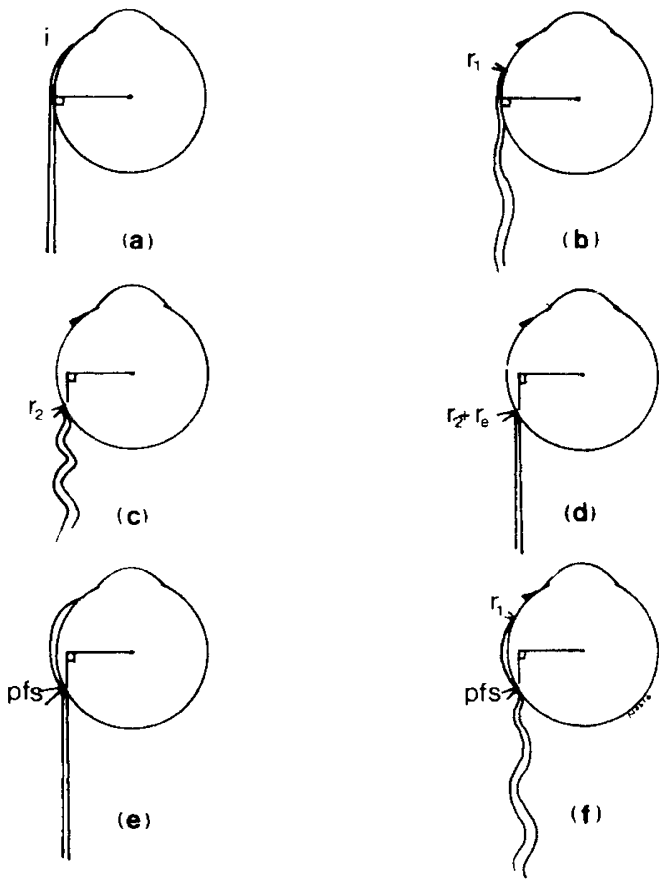

Fig. 1. (a) normal relationship between rectus muscle and eyes. (b) after conventional recession, the muscle is weakened by slackness but still acts through a tangential point on the surface of the globe. (c) after excessive recession the muscle is very slack and action is further weakened as the tangential point of action is no longer at the surface of the globe. (d) if a rectus muscle is both recessed and resected simultaneously by the same amount, there is no slackness but the rotational lever arm is shortened. (e) a faden operation works in the same way. (f) if posterior fixation sutures are combined with a recession, an unusually large effect is produced as all the slackness is taken up by that portion of the muscle between the sutures and its origin at the orbital apex.

$\dagger$ Deceased.

Correspondence to: Department of Ophthalmology General Infirmary at Leeds. 

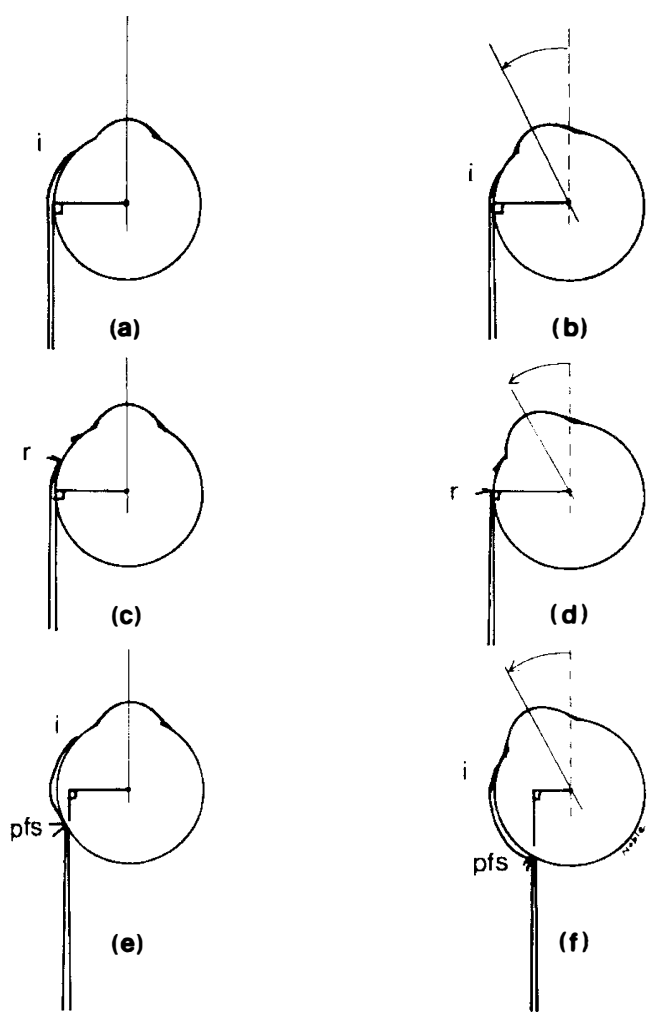

Fig. 2. $(a \& b)$ the normal rectus muscle exerts its action at the surface of the globe both in the primary position and when the eye rotates into its field of action. $(c \& d)$ the same is true after conventional recession. ( $e$ $\& f)$ after application of posterior fixation sutures the power of the operated muscle decreases progressively as the eye moves into its field of action due to increasing shortness of the lever arm of rotation.

effect is achieved by suturing the rectus muscle to the sclera well behind the tangential point of action on the surface of the globe. When the muscle contracts and the eye rotates, the effective length of the lever arm becomes increasingly shorter. ${ }^{2}$ Unless the faden operation is combined with a recession of the muscle insertion, the basic muscle tone remains essentially unchanged because the distance between the muscle's origin and insertion is unaltered. It is as if the muscle had been both recessed and resected by the same amount. If the faden operation is combined with a recession, the effect of the latter is enhanced as all the slackness is taken up in the relatively short portion of the muscle between its origin and the points of insertion of the posterior fixation sutures (Figs. $1 \& 2$ ).

\section{Method}

The posterior fixation sutures must be strong and permanent; 3-0 or 4-0 Dacron or Supramid are commonly used. Because the lengths and orientations of the rectus muscles differ, as do the distances of their insertions from the limbus, the necessary fixation points are also different (Table II). Two different techniques can be employed:

(1) The rectus muscle is attached by its edges to the sclera using whipped sutures, each incorporating some $1 / 5$ of the total muscle width.

(2) The muscle is detached at its insertion. One or two circumferential mattress sutures are then applied to the sclera and through the muscle belly, and the muscle is reinserted.

The second method is more easily combined with recession of the muscle insertion. Proponents of the first technique claim that it has some weakening effect on the muscle even when the eye is in the straightahead position; combination with a recession is not therefore often required. ${ }^{3}$

Table II Approximate position for optimal posterior fixation behind normal rectus muscle insertion.

\begin{tabular}{ll}
\hline Medial rectus & $11-13 \mathrm{~mm}$ \\
Vertical recti & $12-14 \mathrm{~mm}$ \\
Lateral rectus & $17-19 \mathrm{~mm}$ \\
\hline
\end{tabular}

The operation, however performed, probably produces other effects. In particular, the heavy fixation sutures induce quite a large area of scarring which increases the forces resisting rotation of the eye. Another mechanical effect is the holding back of the orbital fat pad behind the fixation sutures. This damps rotation of the eye and it also helps to resist the globe retraction which is threatened by active contraction of a muscle which has been attached to the globe behind its tangential point of contact.

\section{Indications}

A. By making use of the progressive weakening in the field of action of the operated muscle

Because the faden operation, unlike con- 
ventional recession, has a progressive weakening effect when the eye is rotated towards the operated muscle, but does not change the resting position of the eye to any great extent, it can usefully be employed:

(1) to reduce dissociated vertical divergence $(D V D)$. This is probably the most widely acknowledged indication. As the dissociated eye drifts upwards there is a progressive weakening of the elevating force produced by the surgery which can at least diminish the abnormal movement to an extent which makes it cosmetically much less noticeable. The effect is more profound if combined with muscle recession and this is especially necessary when asymmetrical DVD is associated with hypertropia of the non-fixing eye. The immediate effects are usually encouraging, but later recurrence is quite common.

(2) to reduce convergence excess. These patients with accommodative esotropia exhibit, after optical correction of their hypermetropia, no manifest esodeviation on distance fixation but a convergent strabismus for near. If bifocal spectacles are considered inappropriate, sufficient conventional surgery to make their eyes straight for near is then likely to induce either a mechanical impairment of convergence or a consecutive exodeviation for distance. A faden operation on both medial recti does not change the distance angle much but induces a marked weakening of convergence. It may be used as a secondary procedure when previous bilateral medial rectus recessions have failed to control the strabismus. Alternatively, it may be carried out in isolation or combined with bilateral recessions as a primary procedure if there is a significant basic angle of strabismus (esophoria on distance fixation).

(3) to weaken the contralateral synergist in cases of muscle paresis. The consequent progressive weakening of the operated muscle's action as it turns the eye matches that of its contralateral synergist, the paresed muscle, thereby inducing greater concomitance and an increase in the field of binocular single vision.

(4) in the treatment of nystagmus blockage syndrome and essential infantile esotropia. This is a widely used and yet contentious indication for the operation. The rationale according to continental European advocates is that patients with these related disorders exhibit a very variable angle of strabismus due to spasmodic nonaccommodative convergence excess, either as a strategy to block nystagmus or due to a developmental oculomotor defect. The most appropriate operation is therefore one which corrects the basic angle of strabismus (by bilateral medial rectus recession) and inhibits spasmodic convergence (by posterior fixation sutures applied to the same muscles). ${ }^{4}$ Although many advocate this combined procedure, others state that the edge-whipping faden technique without recession gives good results. ${ }^{5}$

B. When a previous maximum conventional recession operation has proved insufficient There are many instances in which a maximum conventional recession operation alone fails to induce adequate desired change in the position of the operated eye. The options of reoperating on the same muscle are then limited. A further direct recession will cause mechanical limitation of movement. Re-recession operations using hang-loose or loop sutures have their advocates, but usually the muscle does not reattach indirectly to the tangential point of rotation on the sclera as desired, but directly and in an uncontrolled fashion where the severed insertion lies in direct contact with the globe; the problems of mechanical restriction again ensue. Similarly, marginal myotomy can lead to extensive adherence of the traumatised muscle belly to the underlying sclera. In these circumstances, posterior fixation sutures whipped to the 
edges of the previously recessed muscle will weaken the lever arm of rotation without inducing that additional muscle slackness which follows a re-recession, and can be the most appropriate and effective procedure.

C. To augment the effect of a conventional recession operation

As already intimated, when a faden operation and recession are combined, less recession of the rectus insertion is required than normal in order to effect a certain alteration in the resting position of the eye as the slackness is all taken up in the short length of the muscle behind the posterior fixation sutures. There is also some weakening due to damage caused to the anterior part of the muscle belly by the posteriorly placed sutures. In the same way, a maximum conventional recession is augmented by posterior fixation sutures applied at the same time. The combined operation will lead to some limitation in ocular movement, but this may be acceptable in extreme gaze positions in certain instances where recession alone gives inadequate results. The most appropriate example of this is in the Kestenbaum operation for congenital nystagmus. It is widely appreciated that very large amounts of rotation of the eyes within the muscle cones are required in order to achieve any marked permanent change in the position of the null zone in such cases, with consequent reduction in the compensatory head posture and increased comfort of vision. In some instances, even a combination of very large 'supramaximal' conventional recession and resection procedures proves insufficient; combining the recessions with posterior fixation sutures then adds an additional weakening effect without significantly increasing the mechanical limitation of movement.

D. To achieve posterior stabilisation of rectus muscle position

The upshoots and downshoots on attempted adduction which occur in some patients with Duane's syndrome have been ascribed to mechanical restriction rather than anomalous innervation. The lateral rectus is tight and the medial rectus may then slip up or down on the surface of the globe when it contracts giving rise to a sudden loss of vertical stability in the position of the eye. Scott ${ }^{2}$ suggested that this could be controlled by posterior fixation sutures applied to the horizontal rectus muscles. It is rather surprising that this logical recommendation has not been very widely taken up. However, the most important consideration is to reduce the tethering effect of the tight lateral rectus. This can be done by dividing the anterior portion of the muscle longitudinally, recessing it and splaying out the two portions to give a horizontal Y-shaped reinsertion. ${ }^{6}$

\section{Problems}

The operation is technically more difficult than conventional recession. The heavy sutures must be inserted well behind the equator without broaching the orbital fat pad, for otherwise fat will prolapse forwards to produce cosmetically unacceptable subconjunctival swelling, discolouration and vascularisation as well as mechanical effects from fibrosis. The vortex veins emerge from the sclera close to the required position of posterior fixation sutures whipped around the edges of the vertical recti. If the alternative mattress suture technique through the muscle belly is used, the sclera is very thin in those submuscular areas; this is a special hazard in view of the heavy gauge permanent suture material which must be used. These sutures themselves induce significant local scarring, and if they do not the operation is probably ineffective in the long-term. This fibrosis means that the operation is not entirely reversible after the immediate post-operative healing period. The surgery is particularly difficult when applied to the superior rectus muscle, usually in the treatment of DVD. Access is hampered by the proximity of the orbital roof and by the vortex veins, but principally by the superior oblique tendon which lies exactly in the area where the posterior fixation sutures need to be inserted. This problem can be dealt with by deflecting the tendinous insertion anteriorly, or less satisfactorily by passing the scleral sutures directly through the tendon. 


\section{Evaluation}

The faden operation is based on an interesting theoretical concept, and its mode of action is indeed different. from that of other rectus muscle weakening operations. It therefore has special applications as outlined. The degree to which it is used depends very much on the experience of the individual surgeon. The technical problems decrease with familiarity, but doubts remain about the longterm durability of the initially satisfactory results obtained. While there is a considerable number of firm adherents to the technique in continental Europe, it has not found a place worldwide in other than its rather special applications. In particular there is no widespread belief that it is more effective in the long-term treatment of infantile esotropia than maximal medial rectus recession operations. Many surgeons would hesitate to apply such heavy gauge permanent suture material far back on the immature infant sclera unless they were completely satisfied that there was no alternative.
I am indebted to my colleague, $\mathrm{Mr}$ Bruce Noble for the illustrations and to my secretary, Miss Anne Gelder for preparation of the manuscript.

\section{References}

${ }^{1}$ Adelstein FE and Cüppers C: Probleme der operativen Schielbehandlung. Bericht Deutsche Ophthalmologische Gesellschaft 1968, 69: 58093.

${ }^{2}$ Scott AB: The faden operation: mechanical effects. Am Orthop J 1984, 27: 44-7.

${ }^{3}$ de Decker W and Haase W: Results of the faden operation in alternating convergent strabismus of the congenital type. In Balen A.Th.M. van \& Houtman WA (eds) Documenta Ophthalmologica Proceedings Series 32, 1982, pp 65-72. Junk, The Hague.

${ }^{4}$ Berard PVM, Mouillac-Gambarelli N, Reydy R: Management of variable angle esotropia. In Mein M. \& Moore S. (eds) Orthoptics, Research and Practice 1981, pp 233-5, Kimpton, London.

${ }^{5}$ de Decker W: The faden operation. When and how to do it. Trans Ophthalmol Soc UK 1981, 101: 264-70.

${ }^{6}$ Rogers GL and Bremer DL: Surgical treatment of the upshoot and downshoot in Duane's retraction syndrome. Ophthalmology 1984, 91: 1380-3. 